\title{
Transesophageal Echocardiography in Bentall Surgery: Hope or Hype?
}

\author{
V. Krishnan ${ }^{1}$ Mohit Prakash ${ }^{1}$ Sanjeev Kumar ${ }^{1}$ \\ Rohit Malhotra $^{1}$ Poonam Malhotra Kapoor ${ }^{1}$ \\ ${ }^{1}$ Department of Cardiac Anaesthesia and Critical Care, All India \\ Institute of Medical Sciences, New Delhi, India \\ Address for correspondence Poonam Malhotra Kapoor, MD, DNB, \\ MNAMS, FISCU (Hony), FIACTA (Hony), FTEE(Hony), Department of \\ Cardiac Anaesthesia and Critical Care, CTC, AlIMS, New Delhi 110029, \\ J Card Crit Care 2021;5:257-262. \\ India (e-mail: drpoonamaiims@gmail.com).
}

\begin{abstract}
Keywords

- Bentall procedure

- intraoperative

- postoperative

- TEE

A 57-year-old male patient who is a known case of hypertension well controlled on ramipril presented with a history of shortness of breath and palpitation of 3 months duration. The symptoms were insidious in onset; however, it progressed rapidly over the period of 3 months, wherein at present the patient feels breathless in performing normal daily activities of living making him New York Heart Association class III patient. Palpitations are regular in nature and present throughout the time and are exacerbated on performing even light activities within his house. There was associated swelling of bilateral lower limbs that usually improved after a night's rest; however, it was not completely resolved. The individual did not give any history of chest pain, presyncope, syncope, or any other symptoms that pointed toward a cardiac condition.
\end{abstract}

\section{Introduction}

The essentiality of having a transesophageal echocardiography (TEE) cover the Bentall surgery is often overlooked.

\section{Case History}

A 57-year-old male patient who is a known case of hypertension well controlled on ramipril presented with a history of shortness of breath and palpitation of 3 months duration. The symptoms were insidious in onset; however, it progressed rapidly over the period of 3 months, wherein at present the patient feels breathless in performing normal daily activities of living making him New York Heart Association class III patient. Palpitations are regular in nature and present throughout the time and are exacerbated on performing even light activities within his house. There was associated swelling of bilateral lower limbs that usually improved after a night's rest; however, it was not completely resolved. The individual did not give any history of chest pain, presyncope, syncope, or any other symptoms that pointed toward a cardiac condition.

Consultation in the cardiology clinic at our institute at All India Institute of Medical Sciences, CTC, New Delhi, was sought. General physical examination revealed a bounding radial pulse with a normal rate and rhythm. The patient had high systolic blood pressure reading $(150-160 \mathrm{~mm} \mathrm{Hg}$ ) and low diastolic blood pressure reading $(40-50 \mathrm{~mm} \mathrm{Hg})$ with a wide pulse pressure. There was bilateral pedal edema till ankle that was pitting in nature. On systemic examination, the cardiac apex was felt in a downward and outward location suggestive of cardiomegaly. On auscultation of the chest, an early diastolic murmur was heard prominently in the aortic area. Routine investigations that included electrocardiogram (ECG), chest X-ray (CXR), TTE, computed tomography (CT) angiography, and blood workup were done.

ECG was normal with a sinus rhythm of around $75 \mathrm{bpm}$ and normal axis. CXR showed a CT ratio $>0.6$, with widened mediastinum and the cardiac apex displaced down and left.
DOI https://doi.org/ $10.1055 / s-0042-1742715$. ISSN 2457-0206. (c) 2022. Official Publication of The Simulation Society (TSS), accredited by International Society of Cardiovascular Ultrasound (ISCU). All rights reserved.

This is an open access article published by Thieme under the terms of the Creative Commons Attribution-NonDerivative-NonCommercial-License, permitting copying and reproduction so long as the original work is given appropriate credit. Contents may not be used for commercial purposes, or adapted, remixed, transformed or built upon. (https://creativecommons.org/ licenses/by-nc-nd/4.0/)

Thieme Medical and Scientific Publishers Pvt. Ltd., A-12, 2nd Floor, Sector 2, Noida-201301 UP, India 


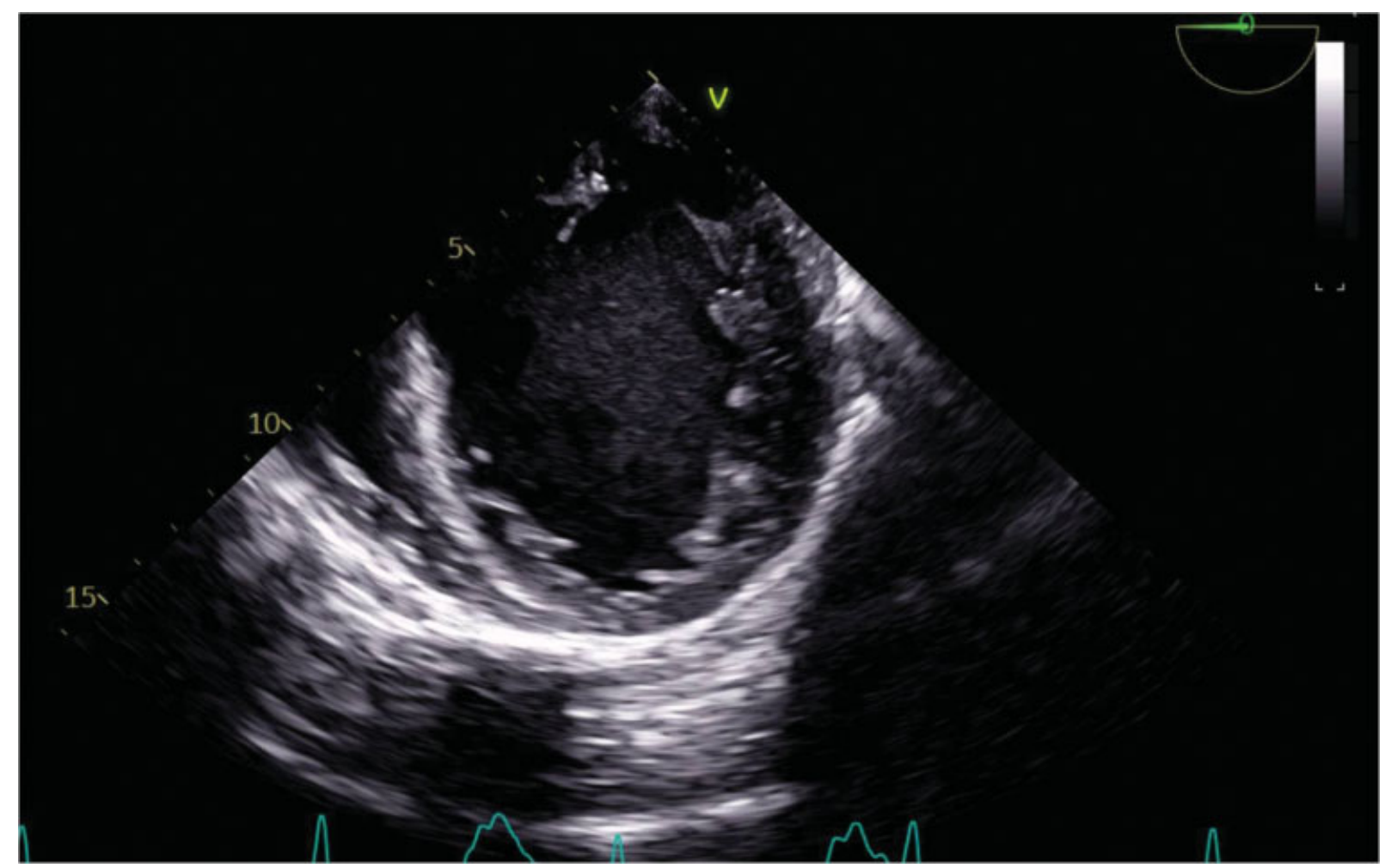

Fig. 1 A two-chamber magnetic resonance view on transesophageal echocardiography showing left ventricular hypertrophy as seen in preoperative period with the left atrium dilated, ejection fraction $<35 \%$, SAV ME showed a tricuspid aortic valve. ME, midesophageal view; SAV, shorts in aortic valve.

\section{Transthoracic ECHO Findings}

Two-dimensional TTE revealed a bicuspid aortic valve with severe aortic regurgitation, with increased dimensions of aortic sinus $(49 \mathrm{~mm})$, sinotubular junction (STJ) $(68 \mathrm{~mm})$, ascending aorta $(96 \mathrm{~mm})$. and a reduced ejection fraction (35-40\%). CT angiography confirmed the findings with the following measurements: annulus $(33 \times 26 \mathrm{~mm})$, sinus $(49 \times 49 \times 49 \mathrm{~mm})$, effacement of STJ, ascending aorta $(103 \mathrm{~mm})$. Coronaries were normal and showed no obstruction to flow.

Blood investigations were within normal range except for deranged serum urea and creatinine $(58 / 1.6 \mathrm{mg} / \mathrm{dL})$ preoperatively. Intraoperative TEE probe was inserted, as is a mandate in all cardiac surgery patients, in our center. The images found are shown from - Figs. 1 to 6 .

\section{Discussion}

The Bentall procedure entails the surgical implantation of a composite graft to replace the aortic valve and portion of the ascending aorta, with the reimplantation of the coronary arteries into the graft. TEE has an invaluable role in the prognosis, especially in detecting the coronary blood flow, post-surgery (-Fig. 7).

Early postoperative TEE may show a periaortic hematoma with markers of inflammation such as fever, which the patient may develop in the intensive care unit. A periaortic abscess leading to fever must be distinguished from a periaortic hematoma both due to surgical manipulations of the graft or due to graft leaking; TEE is the gold standard for any endocarditis detection, with or without any abscess. A TTE can postoperatively detect paraprosthetic regurgitation just as well as a TEE. Strong prosthetic reflectors with anterior washing jets ( - Figs. 7 and 8 ) may actually be color Doppler artifacts that seem to move through the solid structures of the prosthetic root. These are better visualized on TTE than with TEE. ${ }^{1-3}$ TEE estimates the aortic regurgitation volume, pre- and post-Bentall procedure, much better than the TTE does also; TEE is better for paraprosthetic regurgitation in a prosthetic aortic valve and also when aortic regurgitation is eccentric. ${ }^{4}$ There may be high transprosthetic gradients, post-Bentall procedure, or some thrombosis or asymptomatic aortic prosthetic dysfunction, but this is less in aortic valve than in a mitral prosthetic valve (-Fig. 8). Our patient has high aortic gradients as it was a case of severe aortic stenosis as well as severe aortic regurgitation. With passage of time, a surveillance TEE done will show the left ventricular remodeling with better aortic gradients across the left ventricular outflow tract.

\section{Conclusion}

Performing TEE during the Bentall surgery, preoperatively, intraoperatively, and postoperatively, is essential and recommended for all patients for optimal outcomes. TEE should preferably be continued with TTE in the early postoperative period to rule out high gradients for periaortic hematomas and/or abscesses. Performing TEE in all stages of Bentall procedure is a hope, not a hype. 


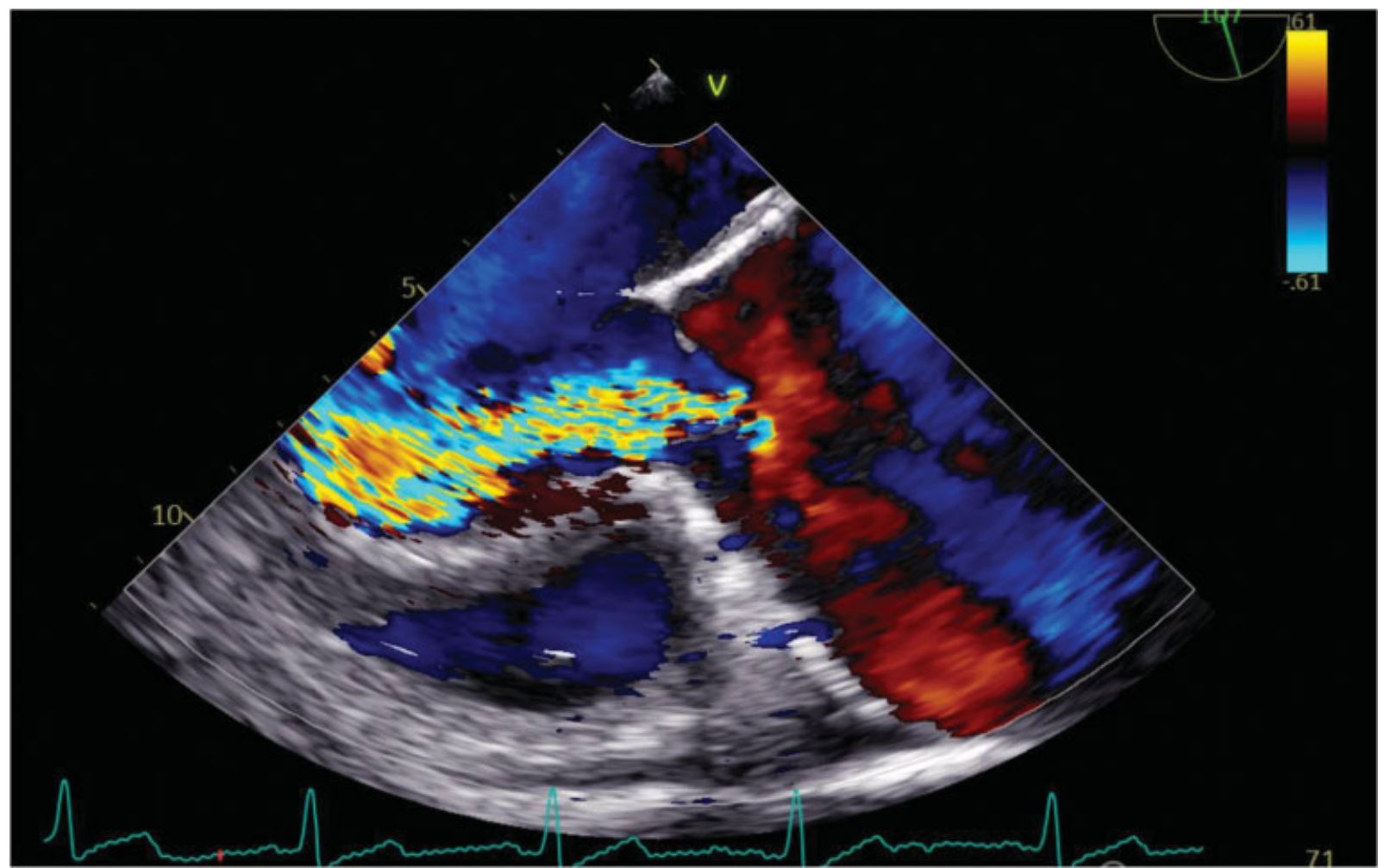

Fig. 2 Preoperative AS and aortic regurgitation (AR) jet seen in the midesophageal long axis view. The AR jet is severe occupying whole of the left ventricular outflow tract with a severe AS as well. AS, aortic stenosis.

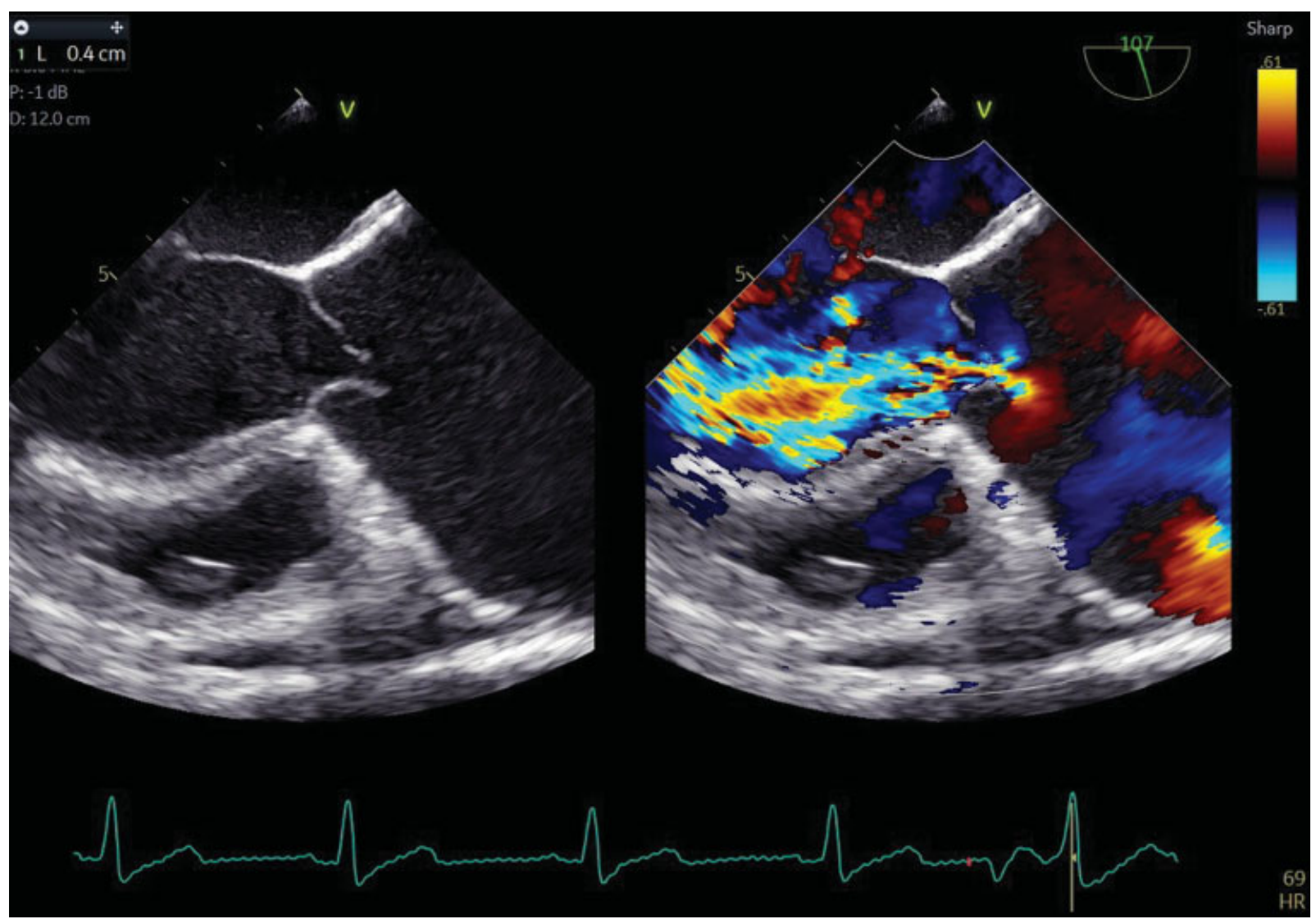

Fig. 3 Preoperative measurement of vena contracta in midesophageal long axis view shows the aortic regurgitation severity as severe. 


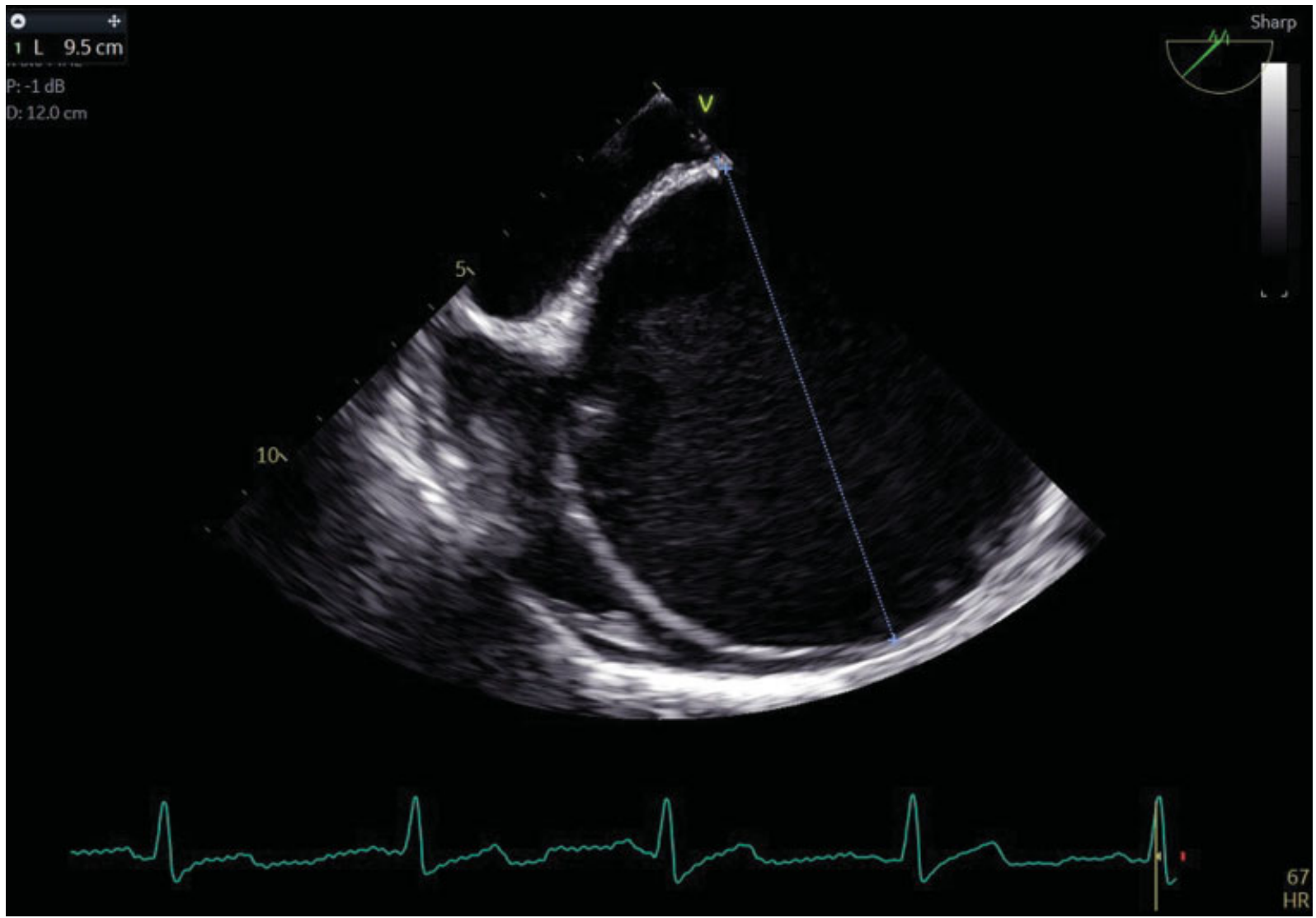

Fig. 4 Preoperative aortic measurement in operative room across the ascending aorta $>5.0 \mathrm{~cm}$, which makes it a candidate for the replacement of the aneurysm small ascending aorta.

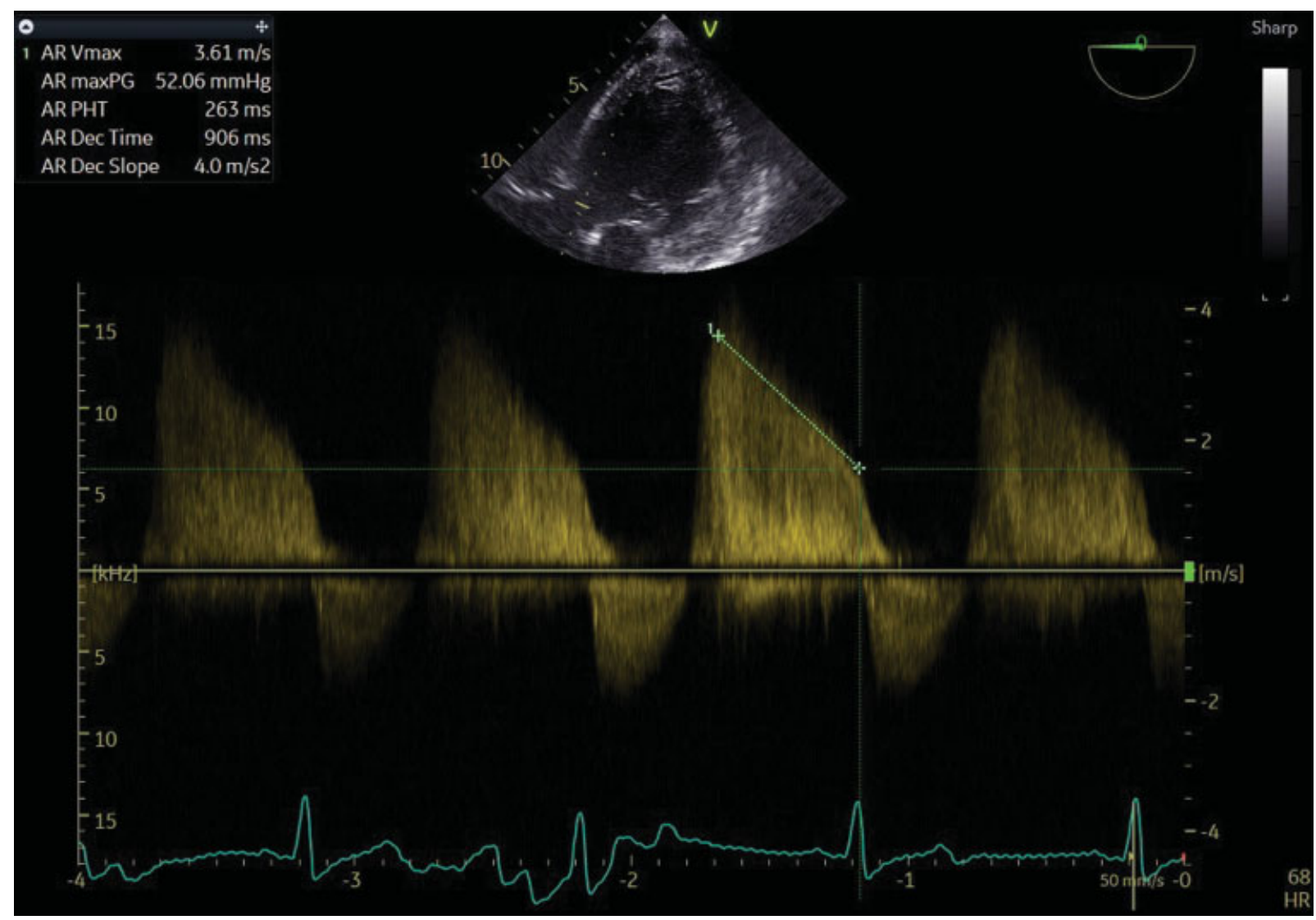

Fig. 5 Preoperative measurement of gradient of aortic regurgitation and AS, showing the gradients both peak and mean as high. 


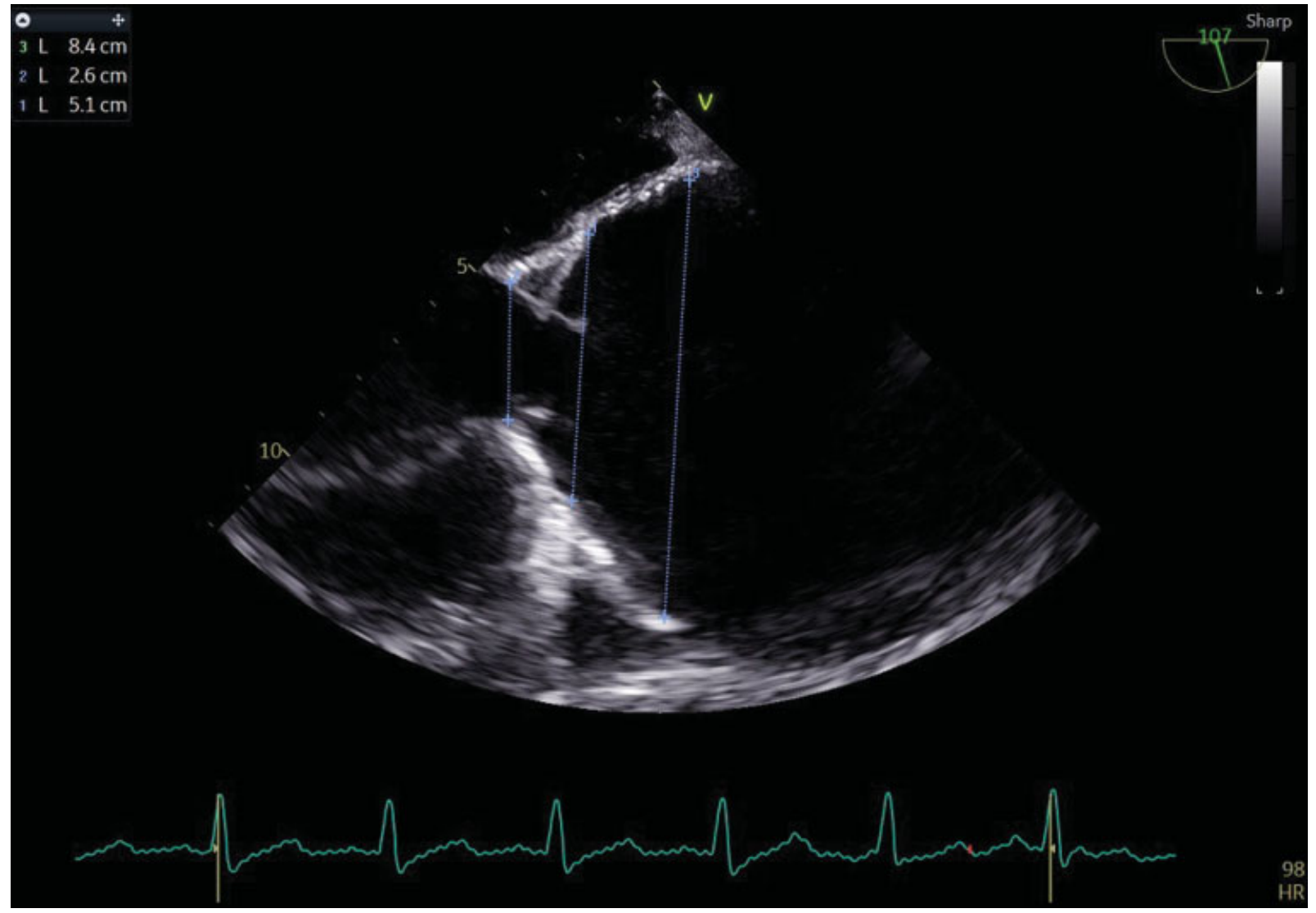

Fig. 6 Preoperative measurement of aortic annulus and aorta in midesophageal long axis view across the aortic annulus, the sinotubular junction, and the ascending aorta.

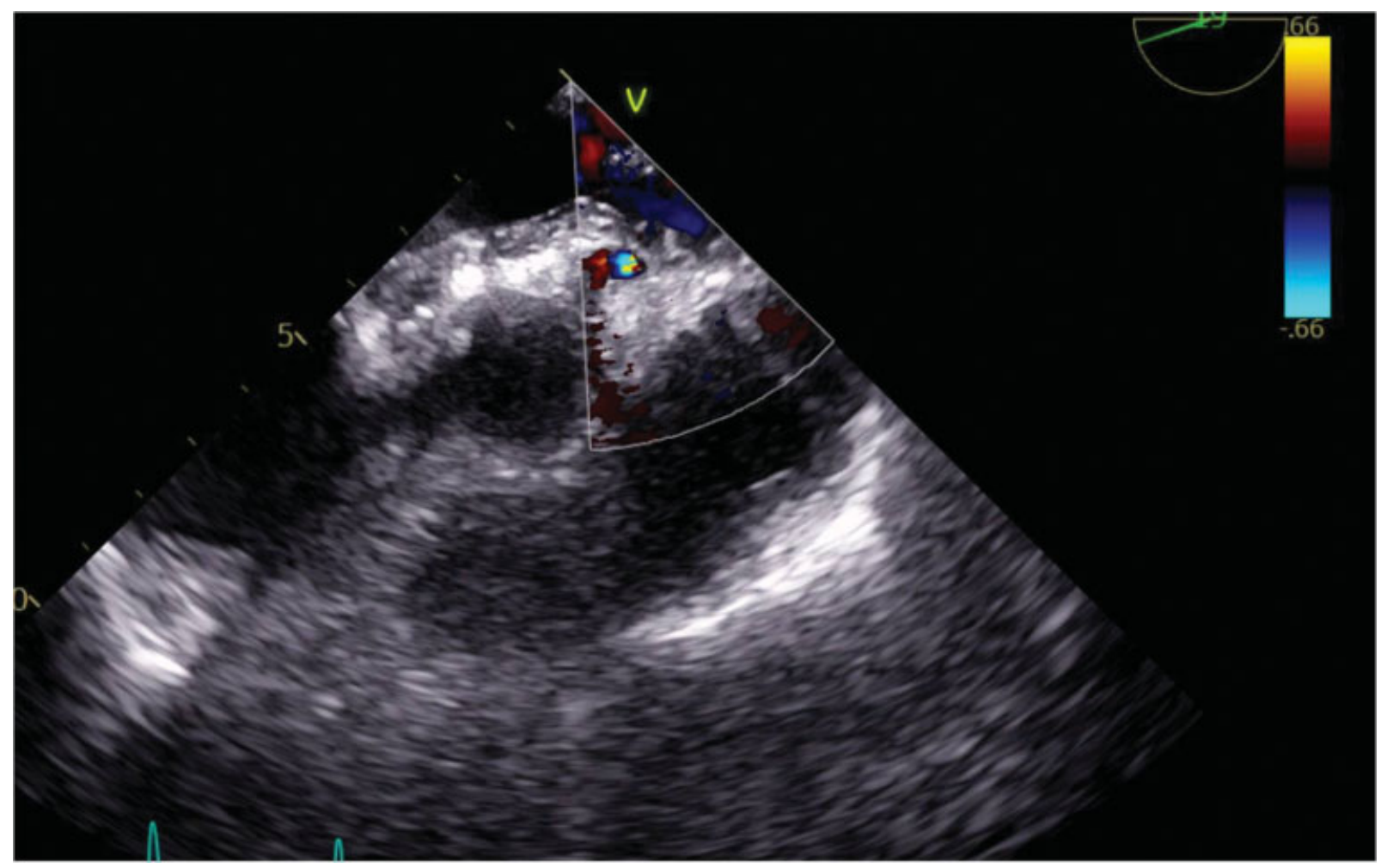

Fig. 7 Coronary blood flow seen on echocardiography in postoperative period, across the left coronary ostia of the aortic valve from RCCC and LCCC. No paraortic hematoma or abscess are detected. RCCC, right coronary cusp commissure; LCCC, left coronary cusp commissure. 


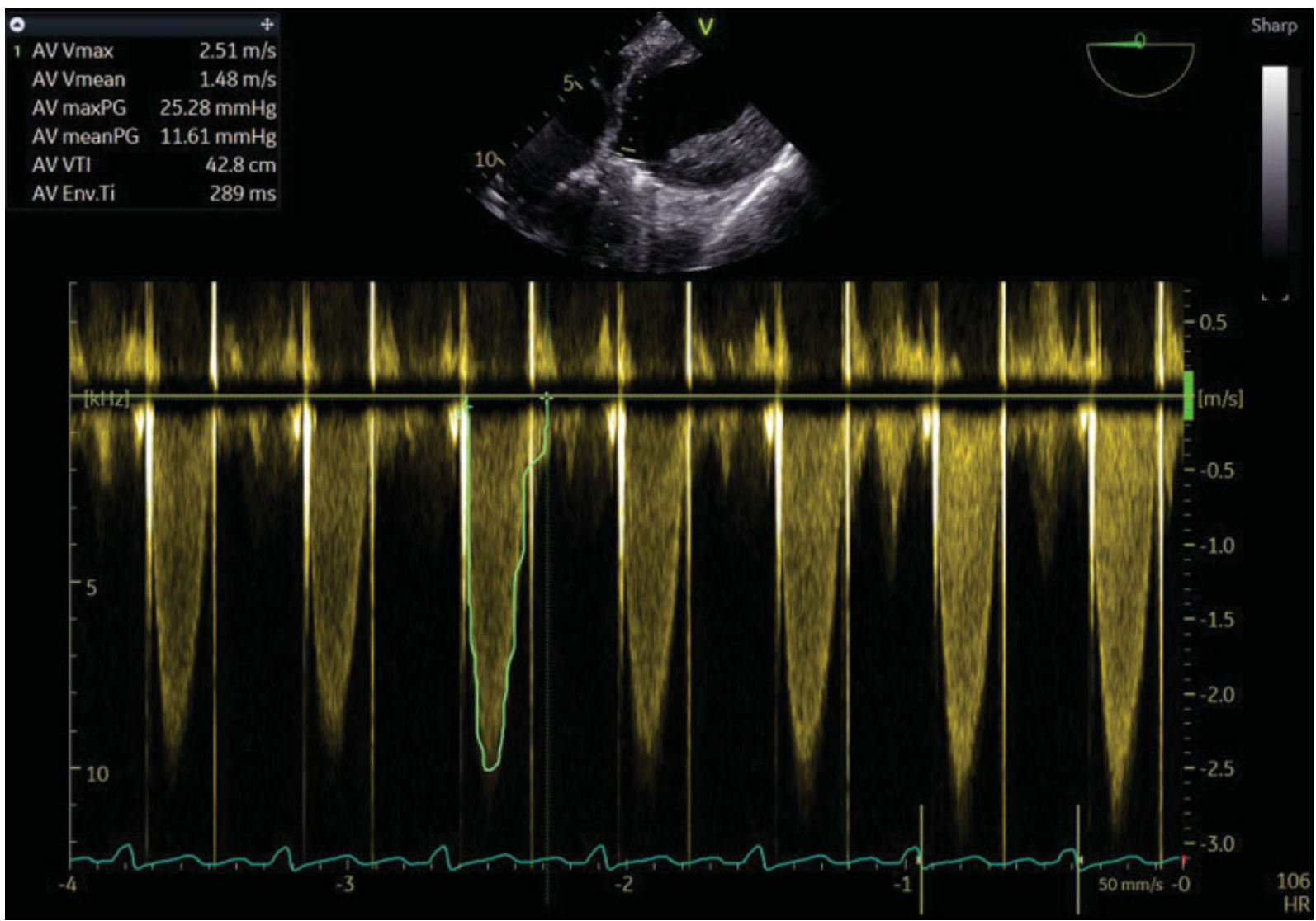

Fig. 8 Postoperative gradient measurement across the aortic valve remains high but shows no aortic regurgitation jets. The prosthetic shadow is showing anterior wash. Strong prosthetic graft reflectors are seen.

\section{Conflict of Interest \\ None declared.}

\section{References}

1 Adam MC, Tribouilloy C, Mirode A, Marek A, Rey JL, Lesbre JP. [Value and limits of single-plane transesophageal echocardiography in dysfunctions of aortic valve prosthesis]. Arch Mal Coeur Vaiss 1993;86(07):1017-1023

2 Niedermeyer J, Daniel WG. [Value of transesophageal echocardiography in diagnosis of diseases of native heart valves]. Herz 1993;18(06):329-340
3 Smith MD, Harrison MR, Pinton R, Kandil H, Kwan OL, DeMaria AN. Regurgitant jet size by transesophageal compared with transthoracic Doppler color flow imaging. Circulation 1991;83 (01):79-86

4 Adam MC, Tribouilloy C, Mirode A, Rey JL, Shen WF, Lesbre JP. [Contribution of transesophageal and transthoracic echography in the evaluation of the mechanism and quantification of regurgitation in mitral and aortic bioprosthetic valves]. Arch Mal Coeur Vaiss 1993;86(09): $1345-1350$ 